\title{
A nanomicelle with miR-34a and doxorubicin reverses the drug resistance of cisplatin in esophageal carcinoma cells by inhibiting SIRT1 signal pathway
}

\author{
Jun Fang ${ }^{1,2 \#}$, Yuezhen Wang ${ }^{1,2 \#}$, Zhun Wang ${ }^{1,2}$, Tieming Xie ${ }^{2,3}$, Fengqin Yan $^{1,2,4}$, Lei Wang ${ }^{1,2,4}$, Jianfeng \\ Hua $^{5}$, Fangzheng Wang ${ }^{1,2,4}$, Zhenfu Fu ${ }^{1,2,4}$, Zhimin Ye ${ }^{1,2,4}$ \\ ${ }^{1}$ Department of Radiation Oncology, Cancer Hospital of the University of Chinese Academy of Sciences (Zhejiang Cancer Hospital), Hangzhou, \\ China; ${ }^{2}$ Institute of Cancer and Basic Medicine (IBMC), Chinese Academy of Sciences, Hangzhou, China; ${ }^{3}$ Department of Radiology, Cancer \\ Hospital of the University of Chinese Academy of Sciences (Zhejiang Cancer Hospital), Hangzhou, China; ${ }^{4}$ Key Laboratory of Head \& Neck Cancer \\ Translational Research of Zhejiang Province, Hangzhou, China; ${ }^{5}$ Department of Radiation Oncology, Quzhou Kecheng Hospital, Quzhou, China \\ Contributions: (I) Conception and design: Z Ye; (II) Administrative support: Y Wang, Z Zhen; (III) Provision of study materials or patients: Z Wang, T \\ Xie; (IV) Collection and assembly of data: F Yan, L Wang; (V) Data analysis and interpretation: J Fang, J Hua; (VI) Manuscript writing: All authors; \\ (VII) Final approval of manuscript: All authors. \\ \#These authors contributed equally to this work. \\ Correspondence to: Zhimin Ye; Zhenfu Fu. Department of Radiation Oncology, Cancer Hospital of the University of Chinese Academy of Sciences \\ (Zhejiang Cancer Hospital), Banshan East Road, Gongshu District, Hangzhou, China; Institute of Cancer and Basic Medicine (IBMC), Chinese \\ Academy of Sciences, Hangzhou, China; Key Laboratory of Head \& Neck Cancer Translational Research of Zhejiang Province, Banshan East Road, \\ Gongshu District, Hangzhou, China. Email: yezm@zjcc.org.cn; fuzf1000@163.com.
}

Background: Esophageal carcinoma (EC) is one of the most deadly malignant tumors in the world. Surgery, combined with chemotherapy or radiotherapy, is the traditional strategy for the treatment of EC. Cisplatin (CDDP) is a common chemotherapy drug widely used to treat EC due to its powerful anti-tumor effect. However, CDDP is subject to intrinsic or acquired resistance in EC cells, which badly hinders the efficacy of chemotherapy. The resistance phenomenon is mostly caused by the p53 mutant in the EC and the low efficiency of the drug delivery system.

Methods: In this study, a specially designed nanomicelle was used to promote the anti-tumor effect of chemotherapy drugs against the CDDP-resistant EC cells. The nanomicelle consisted of miR-34a, doxorubicin (DOX), polyethylene glycol (PEG), and other excipients in an appropriate ratio.

Results: The results showed that the nanomicelle could exert significant cell proliferation inhibition and apoptosis-inducing effects in the CDDP-resistant EC cells. The endogenous expression of miR-34a in the CDDP-resistant EC cells was promoted by the incubation with the nanomicelle. After incubation with the nanomicelle, the expression of protein SIRT1 was inhibited, and the expression of caspase 3 was promoted significantly in the CDDP-resistant EC cells.

Conclusions: Our results indicate that the specially designed nanomicelle can exert promising anti-tumor effects by introducing miR-34a to inhibit SIRT1 signaling pathway and enhance the efficiency of the drug delivery system.

Keywords: Nanomicelle; miR-34a; esophageal carcinoma (EC); p53; SIRT1; drug resistance

Submitted May 26, 2019. Accepted for publication Mar 03, 2020.

doi: $10.21037 /$ tcr-19-975

View this article at: http://dx.doi.org/10.21037/tcr-19-975 


\section{Introduction}

Esophageal carcinoma (EC), known for its high malignancy among cancers, ranks sixth and ninth in global incidence for males and females, respectively (1), and is rapidly threatening the health of citizens worldwide (2). Although patients with EC can undergo a series of therapies, the long-term survival rate of EC remains low $(3,4)$. According to the pathogenesis of $\mathrm{EC}$, it can be divided into two types, with the squamous carcinoma type accounting for approximately $90 \%$ of the total cases (5). Currently, surgery is still the primary therapy for EC (6). However, many patients develop metastatic disease or locoregional recurrence soon after surgery $(7,8)$. As a consequence of this, surgery combined with chemotherapy or radiotherapy is becoming the logical choice for EC treatment (9).

Cisplatin (CDDP), one of the most powerful anticancer chemotherapy drugs, is widely used to treat EC (10). The anti-tumor mechanism of CDDP relies on its ability to combine with DNA, resulting in the formation of CDDPDNA adducts, which can inhibit the replication and transcription of DNA, inducing tumor cell death (11). However, the application of CDDP is considerably limited by the intrinsic or acquired resistance in EC cells (12). As the anti-tumor effect of CDDP, if left unhindered, is significant, creating new approaches or strategies to reverse the resistance and enhance the anti-tumor effect of CDDP is urgently needed.

Recent research suggests that both internal factors and external factors contribute to the resistance and variable response rate to CDDP during the treatment of EC (13). Among the internal factors, $\mathrm{p} 53$ mutant has attracted the most attention in recent years. P53 was one of the first tumor suppressor genes to be discovered and is capable of suppressing tumor cell proliferation by inducing cell cycle arrest and apoptosis in conditions where the DNA is damaged (14). However, in most human tumor types, the p53 mutant is rendered ineffective, and the EC is no exception $(15,16)$. Thus, exploring strategies to enhance the function of $\mathrm{p} 53$ is important for reversing CDDP resistance $(17,18)$. In terms of external factors, the largest concern is the uneven distribution of drugs within the tumor and subcellular fraction (19). The maldevelopment of vessels in the tumor and the low transferring rate of anti-tumor drugs result in the uneven distribution of drugs at the target sites $(20,21)$. To improve the effectiveness of drugs impeded by external factors, new effective drug delivery methods should be developed.

MiR-34a is used to enhance the function of the $\mathrm{p} 53$ pathway and doxorubicin (DOX)-mixed nanomicelles can increase the transferring efficiency of chemotherapy drugs. Thus, with the aim to overcome the drug resistance of chemotherapy precipitated by both internal factors and external factors, a new drug package containing miR-34a and DOX-mixed nanomicelles was constructed.

\section{Methods}

\section{Human esophagus squamous cancer cell lines}

KYSE-410, an esophageal squamous cancer cell (ESCC) line found in humans and reported to have the p53 mutant, was acquired from BoGu Biological Science and Technology Co., Ltd. (Shanghai, China). The cells were subjected to culture with RMPI medium 1640 containing $10 \%$ fetal bovine serum, $100 \mathrm{U} / \mathrm{mL}$ penicillin, and $100 \mu \mathrm{g}$ streptomycin. This was followed by incubation at $37^{\circ} \mathrm{C}$ in a $5 \% \mathrm{CO}_{2}$ humidified incubator.

\section{MTT assay for assessing cell growth}

Cell viability was detected by MTT assay (Sigma, MO, USA) as per the protocol of the sourcing company. Briefly, the cells were seeded in a 96-well plate for overnight incubation. CDDP in doses varying from 0.25 to $8 \mu \mathrm{g} / \mathrm{mL}$ were added to the cells. After incubating for $72 \mathrm{~h}$, MTT $(0.5 \mathrm{mg} / \mathrm{mL})$ was added, and the cells underwent $4 \mathrm{~h}$ of further incubation. The formazan precipitate was dissolved in about $200 \mu \mathrm{L}$ of dimethyl sulfoxide (DMSO), and at $490 \mathrm{~nm}$, the absorbance was assessed by a Benchmark microplate reader (Bio-Rad, CA, USA). This assay procedure was independently repeated 3 times. The cell growth inhibition rate was calculated as follows: inhibition rate $=\left(\mathrm{OD}_{\text {control }}-\mathrm{OD}_{\text {treatment }}\right) / \mathrm{OD}_{\text {control }}$.

\section{The construction of the CDDP-resistant strains (KYSE- 410-CisR)}

First, the MTT assay was used to calculate the $\mathrm{IC}_{50}$ of CDDP in KYSE-410 cells. The cells in the logarithmic phase were digested by pancreatin and treated as a singlecell suspension. The number of cells was adjusted to 

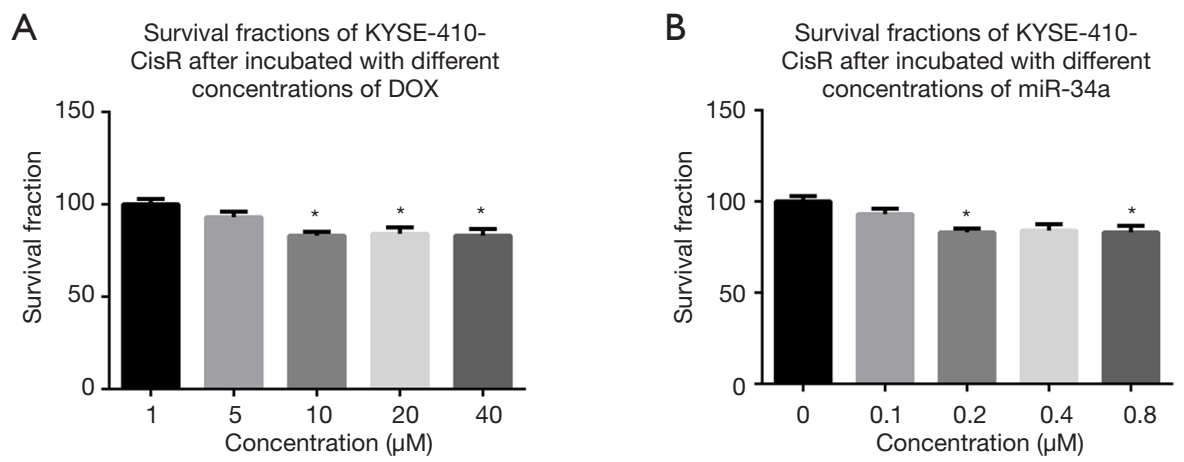

Figure 1 The construction of CDDP-resistant strains-KYSE-410-CisR. (A) The survival fraction of KYSE-410-CisR after incubation for $48 \mathrm{~h}$ with $1,5,10,20$, and $40 \mu \mathrm{M}$ DOX; (B) the survival fraction of KYSE-410-CisR after incubation for $48 \mathrm{~h}$ with $0,0.1,0.2,0.4$, and $0.8 \mu \mathrm{M}$ miR-34a mimic ${ }^{*}, \mathrm{P}<0.05$ vs. $\left.0 \mu \mathrm{M}\right)$. DOX, doxorubicin.

$1 \times 10^{5} / \mathrm{mL}$. Approximately $3 \mathrm{~mL}$ of single cell suspension was added into the flask and incubated at $37{ }^{\circ} \mathrm{C}$ in a humidified incubator with $5 \% \mathrm{CO}_{2}$. Two days later, the medium was removed and the flask rinsed twice with phosphate buffer saline (PBS) to remove the dead cells. A further $3 \mathrm{~mL}$ of flesh medium and a certain concentration of CDDP (according to the $\mathrm{IC}_{50}$ of CDDP) were added to the flask which was incubated for $24 \mathrm{~h}$. The medium containing CDDP was removed and the flask had blank medium (without CDDP) added to it. The cells were incubated for 24-48 h; most cells were apoptotic with only a few cells surviving. The CDDP had been added again in the same fashion as the above when the surviving cells went through the logarithmic phase. The whole procedure lasted approximately 2 months, and the treated cells were tested by MTT again to calculate the $\mathrm{IC}_{50}$ of CDDP. The two values of $\mathrm{IC}_{50}$ were compared to determine if the CDDPresistant strains were successfully constructed.

\section{The construction of nanomicelle}

The miR-34a mimic, which can enhance the biological function of endogenous miR-34a, was synthesized by a chemical method and linked with a N-succinimidyl-3-(2-pyridyldithiol)propionate (SPDP). The sequence for miR-34a mimic is 5 '-UGGCAGUGUCUUAGCUGGUUGU-3' and 5'-ACAACCAGCUAAGACACUGCCA-3'. A mixture of DMSO and chloroform-containing polyethylene-SH (PE-SH) was added into the SPDP-miR-34a solution. The mixture was shocked for $48 \mathrm{~h}$ at room temperature and redundant $\mathrm{PE}-\mathrm{SH}$ was removed from the mixture by chromatography. The mixture was subsequently centrifuged at $12,000 \mathrm{rpm}$ and $4{ }^{\circ} \mathrm{C}$ for $1 \mathrm{~min}$. The supernatant was collected and a mixture of miR-34a-S-S-PE was achieved. Another mixture, containing polyethylene glycol (PEG), a cleave $(\mathrm{CLV})$ with high affinity to matrix metalloproteinase 2 (MMP2), and DOX, was added into the miR-34a-S$\mathrm{S}-\mathrm{PE}$ solution. The solution was mixed by vortex and poured slowly into a beaker containing sterile water. Lastly, a transcriptional activator (TAT)-PEG ${ }_{1 \mathrm{k}}-\mathrm{PE}$ complex, consisting of a cysteine-modified TAT protein, $\mathrm{PEG}_{1 \mathrm{k}}$, and PE, was added. The mixture was stirred for $30 \mathrm{~min}$, and the organic solvent was removed by rotary evaporator. The nanomicelle was formed by filtrating the solution with a millipore filter. The particle size was measured by transmission electron microscopy (TEM; EM-2CX; JEOL Ltd., Tokyo, Japan).

\section{CCK8 assay for accessing proliferation of cells treated with different drugs}

To obtain the most effective concentration of DOX and miR-34a mimic for the inhibition of the proliferation of KYSE-410-CisR, 5 different concentrations for DOX and miR-34a mimic were used to perform CCK8 assay on KYSE-410-CisR. The concentrations for DOX were $1,5,10,20$, and $40 \mu \mathrm{M}$. The concentrations for miR-34a mimic were $0,0.1,0.2,0.4$, and $0.8 \mu \mathrm{M}$. The incubation time was $48 \mathrm{~h}$ for both DOX and miR-34a mimic. As shown in Figure $1 A, B, 10 \mu \mathrm{M}$ was chosen for DOX, and $0.2 \mu \mathrm{M}$ was chosen for miR-34a mimic. The concentration of nanomicelle was a mixture of DOX and miR-34a mimic, the concentrations of which were 10 and $0.2 \mu \mathrm{M}$, 
respectively.

Five groups were created according to the different drugs added into KYSE-410-CisR: (I) control, (II) CDDP $\left(\mathrm{IC}_{50}\right)$, (III) free DOX $(10 \mu \mathrm{M})$, (IV) $0.2 \mu \mathrm{M}$ miR-34a mimic combined with $10 \mu \mathrm{M}$ DOX (miR-34a + DOX), (V) nanomicelle (containing $10 \mu \mathrm{M}$ DOX and $0.2 \mu \mathrm{M}$ miR-34a mimic). After KYSE-410-CisRs were incubated with different drugs for 24,48 , and 72 h, CCK 8 assay (CCK8, Sigma) was used to test the proliferation of the five treated KYSE-410-CisRs according to the manufacturer's instructions. Briefly, the cells were seeded in a 96-well plate at a $10^{4}-10^{5}$ cells/well density in $100 \mu \mathrm{L}$ of culture medium, followed by incubation for $24 \mathrm{~h}$ at $37^{\circ} \mathrm{C}$ in a $5 \% \mathrm{CO}_{2}$ humidified incubator. Then, $10 \mu \mathrm{L}$ of various concentrations of drugs were added into the plate, and the treated cells were incubated for the appropriate length of time (e.g., 24, 48, and $72 \mathrm{~h}$ ). Next, $10 \mu \mathrm{L}$ of CCK8 solution was added into each well of the plate using a repeating pipettor. The plate was incubated for 1-4 h and at $450 \mathrm{~nm}$, the absorbance was assessed by a Benchmark microplate reader (Bio-Rad, CA, USA). This assay procedure was independently repeated 3 times. The survival fraction was calculated as follows: inhibition rate $=\left(\mathrm{OD}_{\text {control }}-\mathrm{OD}_{\text {drugs }}\right) /$ $\mathrm{OD}_{\text {control. }}$.

\section{Flow cytometry for testing the apoptosis of cells treated with different drugs}

The cells were incubated for $24 \mathrm{~h}$ after being mixed with different drugs and were collected into $1.5 \mathrm{~mL}$ tubes. Each tube was added with $10 \mu \mathrm{L}$ fluorescently labeled Annexin V reagent and $5 \mu \mathrm{L}$ PI reagent. Each tube was incubated for $10 \mathrm{~min}$ at room temperature. Approximately $200 \mu \mathrm{L}$ cells were added into the flow tube containing $2 \mathrm{~mL}$ PBS and tested by flow cytometry (BD). Three independent assays were performed.

\section{Isolation of RNA and real-time polymerase chain reaction (RT-PCR) for miR-34a}

The cells were incubated for $24 \mathrm{~h}$ after being mixed with different drugs. Total RNA was collected from the five treated cell lines using a Takara MiniBEST Universal RNA Extraction Kit (Takara, Dalian, China), as per the sourcing company's instructions. Quantification of cells was performed with a NanoDrop spectrophotometer (NanoDrop Technologies, Wilmington, DE, USA). Complementary DNA was reverse-transcribed with a specific RT primer (miR-34a: 5'-GTCGTATCC AGTGCAGGGTCCGAGGTATTCGCACTGG ATACGACACAACC-3', U6 as an internal control: 5'-TGGTGTCGTGGAGTCG-3'). The RT-PCR primers for mature miR-34a or U6 were designed as follows: miR-34a F: 5'-GGTGTGGGCTGGCAGTGTCTT-3' and R: 5'-CCAGTGCAGGGTCCGAGGTAT-3'; U6 F: 5'-CTCGCTTCGGCAGCACA-3'and R: 5'-AACGCTTCACGAATTTGCGT-3'. RT-PCR was conducted with SYBR Premix Ex Taq ${ }^{\text {TM }}$ (Tli RNaseH plus) (Takara, Dalian, China) with an Applied Bio-Rad CFX96 SequenceDetection system (Applied Biosystems). The expression level of miR-34a was defined from the threshold cycle $(\mathrm{Ct})$, with the relative expression levels being calculated using the $2^{-\Delta \Delta C \mathrm{Ct}}$ method after normalization with reference to the expression of U6 small nuclear RNA. Three independent assays were performed.

\section{Western blot assay}

The KYSE-410-CisRs were incubated for $24 \mathrm{~h}$ after being mixed with different drugs, and proteins were isolated from each cell line using the Nuclear and Cytoplasmic Protein Extraction Kit (Beyotime, China). Approximately $40 \mu \mathrm{g}$ of protein was separated on $12 \%$ SDS-polyacrylamide gel (SDS-PAGE), and the gel was placed on a polyvinylidene difluoride (PVDF) membrane (Millipore, MIT, MA, USA). The membrane was blocked with $5 \%$ nonfat dry milk in Tris-buffered saline/0.1\% Tween-20 (TBST) ( $\mathrm{pH}$ 7.4) for $1 \mathrm{~h}$ at room temperature and followed by overnight incubation with primary rabbit anti-human antibodies to Sirt1 (1:1,000), p53 (1:1,000), p21 (1:1,000), caspase3 (1:1,000), and GAPDH (1:1,000) (Abcam, USA). A horseradish peroxidase-conjugated antibody against rabbit IgG (1:5,000, Abcam, USA) was used as a secondary antibody. Incubation of the blots was completed with ECL reagents (Beyetime, China), while protein expression detection was completed by exposure to Tanon 5200-multi. Three independent assays were performed.

\section{Statistical analysis}

Differences of statistical significance were evaluated by oneway analysis of variance (ANOVA) for continuous variables, while the least significant difference (LSD) test was used for the normally distributed data. All analyses were performed in GraphPad Prism 5 software. A P value $<0.05$ was used to determine if differences had statistical significance. 

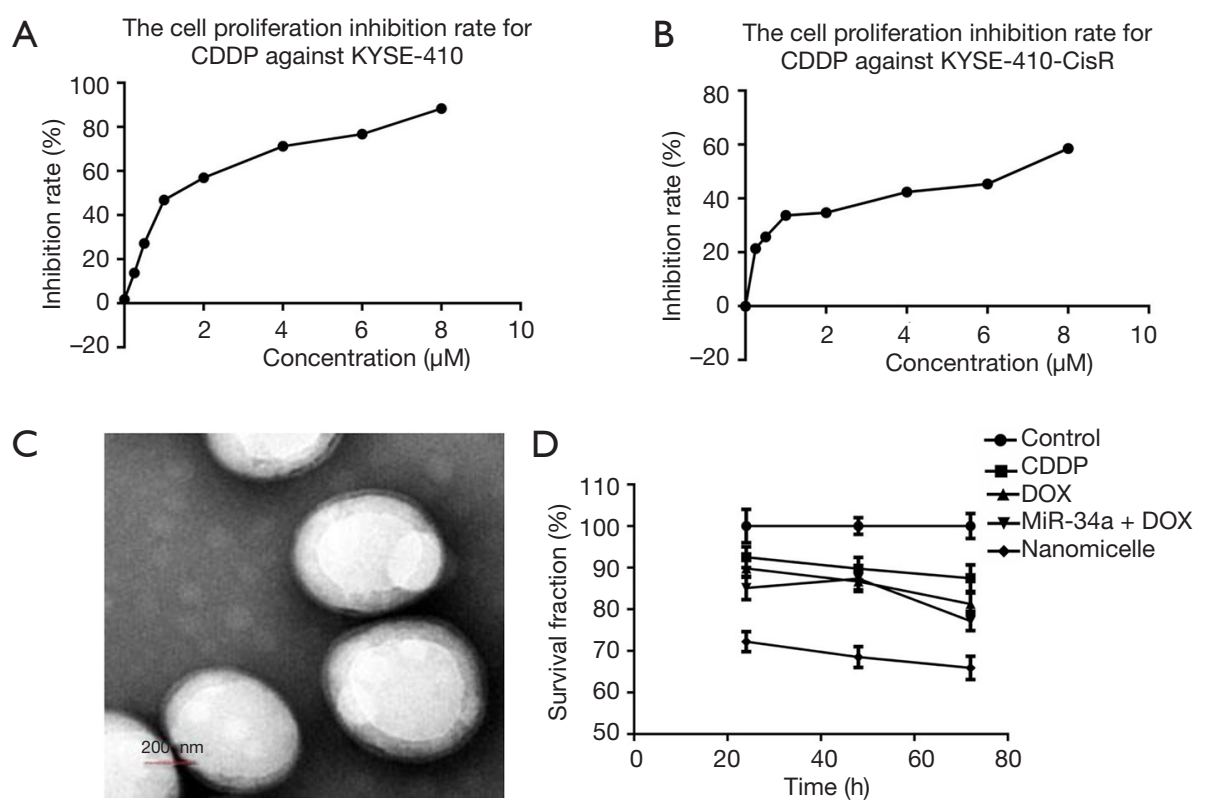

Figure 2 KYSE-410-CisR showed lower survival fraction after dosed with Nano-micelle. (A) The inhibition rate of KYSE-410 proliferation after incubation with different concentrations of CDDP; (B) the inhibition rate of KYSE-410-CisR proliferation after incubation with different concentrations of CDDP. Three independent assays were performed; (C) the morphology of nanomicelle measured by TEM; (D) the survival fraction of KYSE-410-CisR after incubation for 24, 48, and $72 \mathrm{~h}$ with CDDP $(6.524 \mu \mathrm{g} / \mathrm{mL}), \mathrm{DOX}(10 \mu \mathrm{M}), \mathrm{miR}-34 \mathrm{a}(0.2 \mu \mathrm{M})+$ DOX $(10 \mu \mathrm{M}$ ), and nanomicelle (containing $10 \mu \mathrm{M}$ DOX and $0.2 \mu \mathrm{M}$ miR-34a mimic), respectively. CDDP, cisplatin; TEM, transmission electron microscopy; DOX, doxorubicin.

\section{Results}

\section{The construction of CDDP-resistant strains-KYSE-410- CisR}

The $\mathrm{IC}_{50}$ for the inhibition effect of CDDP on KYSE410 was tested by MTT assay. As shown in Figure $2 A$, the inhibition rate increased greatly as the concentration of CDDP increased from 0.25 to $1 \mu \mathrm{g} / \mathrm{mL}$ and plateaued when the concentration of CDDP was more than $4 \mu \mathrm{g} / \mathrm{mL}$. According to the inhibition curve, the value of $\mathrm{IC}_{50}$ was calculated as $1.391 \mu \mathrm{g} / \mathrm{mL}$. After repeated addition of CDDP into the medium of KYSE-410, the $\mathrm{IC}_{50}$ for the inhibition effect of CDDP on the treated cells was tested by MTT assay again. The inhibition curve is shown in Figure 2B. The inhibition rate increased greatly as the concentration of CDDP increased from 0.25 to $2 \mu \mathrm{g} / \mathrm{mL}$ and plateaued when the concentration of CDDP was greater than $4 \mu \mathrm{g} / \mathrm{mL}$. Based on the inhibition curve, the value of $\mathrm{IC}_{50}$ was calculated as $6.524 \mu \mathrm{g} / \mathrm{mL}$ for the inhibition effect of CDDP on treated cells, which is 4.69-fold of that for the inhibition effect of CDDP on KYSE-410. The results confirmed the construction of the CDDP-resistant strains, which were subsequently named KYSE-410-CisR.

\section{KYSE-410-CisR showed lower survival fraction after dosed with nanomicelle}

The TEM image of the nanomicelle stained with phosphotungstic acid on a carbon-coated copper mesh is shown in Figure 2C. The monomolecular micelles were spherical and had a diameter between 100 to $200 \mathrm{~nm}$. The TEM analysis suggested that the nanomicelle size distribution was relatively narrow.

As described in the materials and method section, the five following groups were defined according to the different drugs added to KYSE-410-CisR: control, CDDP, DOX, miR-34a + DOX, and nanomicelle. The results of the proliferation inhibition effect of the four drugs on KYSE410-CisR tested by CCK8 assay are shown in Figure 2D. The survival fraction for the control group was set at $100 \%$. After incubation with CDDP for 24, 48, and $72 \mathrm{~h}$, KYSE410-CisR showed a survival fraction of $92.53 \%, 89.77 \%$, and $89.48 \%$, respectively. After incubation with DOX for 24,48 , and 72 h, KYSE-410-CisR showed a survival 

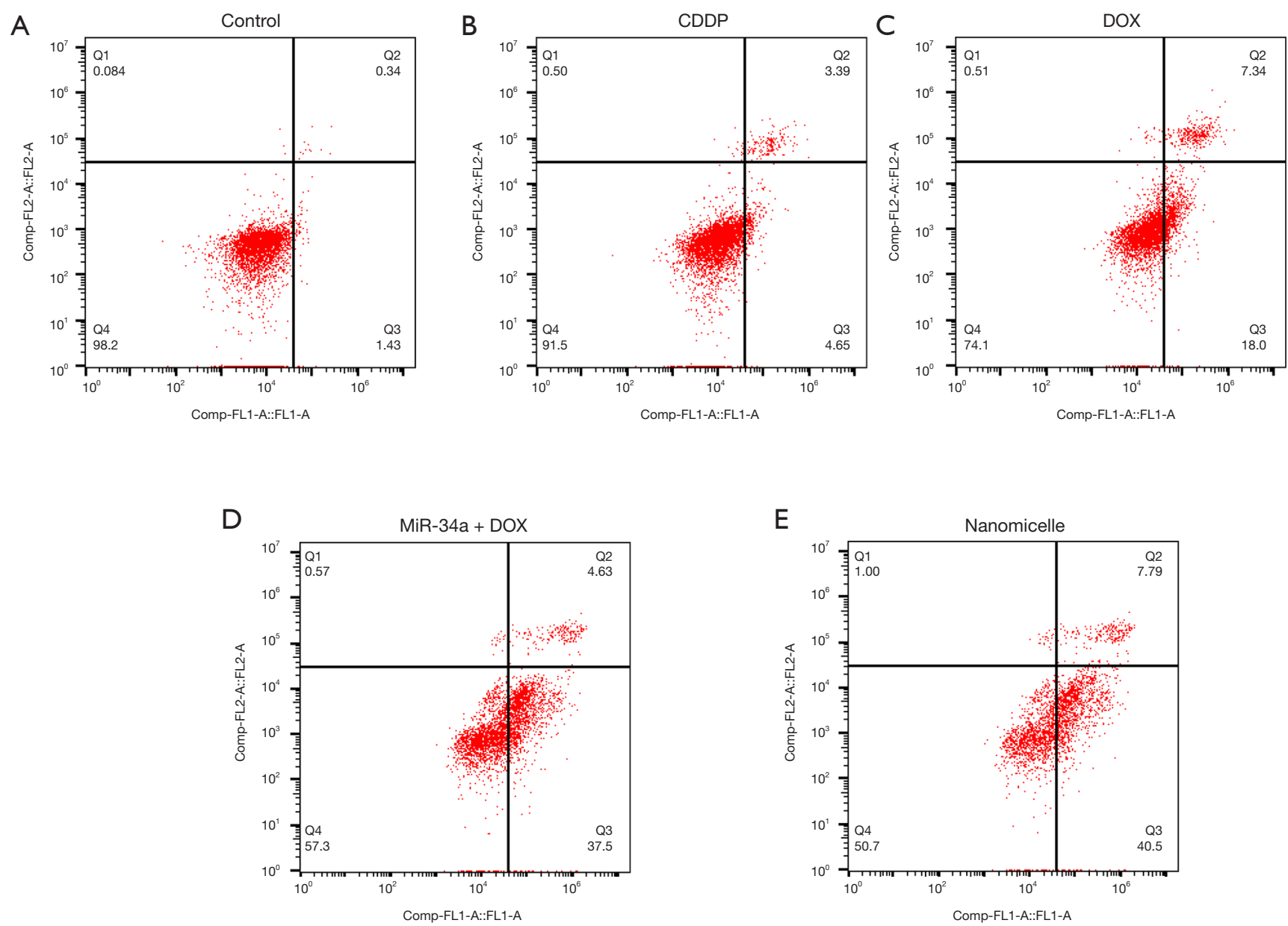

Figure 3 The apoptotic cell percentage was determined using Annexin V-Light 650 reagents in KYSE-410-CisRs after incubation for 24 h with different drugs by flow cytometry. (A) Control; (B) CDDP $(6.524 \mu \mathrm{g} / \mathrm{mL})$; (C) DOX (10 $\mu \mathrm{M})$; (D) miR-34a (0.2 $\mu \mathrm{M})+\mathrm{DOX}(10 \mu \mathrm{M})$; (E) Nanomicelle (containing $10 \mu \mathrm{M}$ DOX and $0.2 \mu \mathrm{M}$ miR-34a mimic). Three independent assays were performed. CDDP, cisplatin; DOX, doxorubicin.

fraction of $89.81 \%, 86.68 \%$, and $81.85 \%$, respectively. After incubation with miR-34a + DOX for 24, 48, and $72 \mathrm{~h}, \mathrm{KYSE}-410-\mathrm{CisR}$ showed a survival fraction of $85.11 \%, 87.38 \%$, and $77.19 \%$, respectively. After incubation with nanomicelle for 24, 48, and 72 h, KYSE-410-CisR showed a survival fraction of $72.21 \%, 68.53 \%$, and $65.91 \%$, respectively. The results indicate that compared with other groups, KYSE-410-CisR showed a lower survival fraction after being incubated with nanomicelle.

\section{Nanomicelle showed the strongest effect on inducing the apoptosis of KYSE-410-CisR}

The effect on inducing the apoptosis of KYSE-410CisR was tested by flow cytometry. As shown in
Figure 3, the fraction of apoptosis of KYSE-410CisR induced by CDDP, DOX, miR-34a + DOX, and nanomicelle was $8.04 \%, 25.34 \%, 42.13 \%$, and $48.29 \%$, respectively. The results indicate that nanomicelle had the strongest effect on inducing the apoptosis of KYSE-410CisR among the five groups.

\section{Nanomicelle increased the expression of SIRT1 by promoting the concentration of miR-34a in KYSE-410- CisR}

After incubating $24 \mathrm{~h}$ with four different drugs, KYSE410-CisRs were lysed to test the expression of miR34a by quantitative PCR (qPCR) assay. As shown in 
The relative expression level of miR-34a

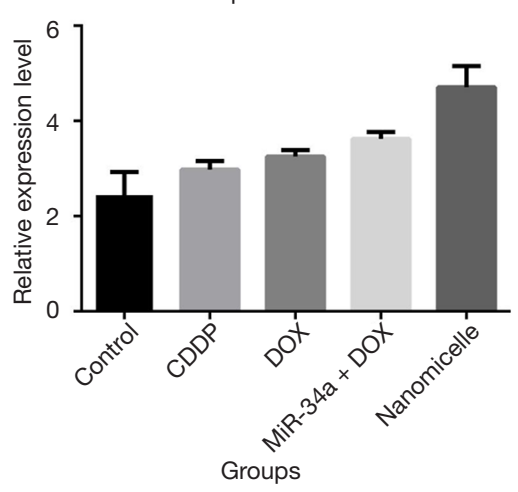

Figure 4 The relative expression level of miR-34a in KYSE-410CisRs after incubation for $24 \mathrm{~h}$ with CDDP $(6.524 \mu \mathrm{g} / \mathrm{mL})$, DOX $(10 \mu \mathrm{M})$, miR-34a $(0.2 \mu \mathrm{M})+\operatorname{DOX}(10 \mu \mathrm{M})$, and nanomicelle (containing $10 \mu \mathrm{M}$ DOX and $0.2 \mu \mathrm{M}$ miR-34a mimic), respectively, detected by qRT-PCR. Three independent assays were performed (*, $\mathrm{P}<0.05$ vs. control; **, $\mathrm{P}<0.01$ vs. control). CDDP, cisplatin; DOX, doxorubicin; qRT-PCR, quantitative real-time polymerase chain reaction.

Figure 4, the expression of miR-34a in the nanomicelle group was higher than that in the control group, with a very significant difference. The expression of miR-34a in the miR-34a + DOX group was relatively lower than that in the nanomicelle group. KYSE-410-CisRs were lysed to test the expression of related proteins by western blotting after incubation for $24 \mathrm{~h}$ with four different drugs. The results of western blot (Figure $5 A, B$ ) showed that the expression of caspase3 in KYSE-410-CisR was significantly promoted by adding nanomicelle, when compared with the control. The expression of SIRT1 in the nanomicelle group was significantly lower than that in the control group (Figure 5C). However, there were no significant differences in the expression of $553 / \mathrm{p} 21$ (Figure $5 D, E$ ) between the nanomicelle group and the control group. The same results were observed in the CDDP, DOX, and miR-34a + DOX groups. These findings suggest that the dose of nanomicelle could increase the expression of SIRT1, but not p53/p21, by promoting the concentration of miR-34a in KYSE-410CisR.

\section{Discussion}

EC is a common digestive tract cancer that occurs among the elderly, with the mortality rate of EC in China being the highest in the world. Chemotherapy is the first choice for the treatment of EC (22). However, many factors may impact the therapeutic effects of chemotherapy drugs, such as the p53 background and the efficiency of drug delivery (23). It is thus necessary to innovate different strategies to overcome these challenges. This study focused on KYSE410 , as it is reported to harbor mutations in the p53 gene, which is itself regarded as the internal factor that hinders the therapeutic effects of chemotherapy drugs. miR-34a was introduced in this study to nullify the p 53 mutant effect. In addition, an improved drug delivery system designed to increase the efficiency of drugs, reaching the effective target sites were also tested.

As an important anti-tumor molecule, the expression level of p53 protein is regulated by many transcription factors, including miR-34a (24). MiR-34a is a recently discovered non-coding RNA that can inhibit the oncogenesis, tumor metastasis, tumor invasion, and prevent drug resistance in chemotherapy (25). The expression level of miR-34a in tumor cells is relatively low, and miR34a can combine with RNA-induced silencing complex (RISC) to regulate the function of the p53 pathway, which mediates the regulation of cell circle arrest, DNA damage repair, apoptosis, and cell aging (26,27). However, as the research into miR-34a progresses, the facts surrounding the anti-tumor effect of miR-34a appear to be more complex than previously thought. Recent reports indicate that miR-34a can increase the sensitivity of CDDP to EC cells by inhibiting the expression of SIRT1, which also participates in the regulation of cell cycle arrest, apoptosis, and cell aging, whereas the expression of p53 remains unchanged (28). In this study, by introducing miR-34a, the cell proliferation inhibition rate and apoptosis fraction of KYSE-410-CisR was significantly improved. In addition, the introduction of DOX and the nanomicelle delivery system could increase the expression level of miR-34a. According to the results of western blot, the anti-tumor effect of miR-34a was mainly associated with the inhibition of SIRT1, but not the regulation of the anti-tumor effect of $\mathrm{p} 53 / \mathrm{p} 21$. As a result, the expression of caspase3, which is apoptosis-related protein, was significantly improved.

It has been reported that the delivery system of nanomicelle has a higher drug-loading rate, drug-loading range, and longer in-vivo circulating interval. Nanomicelle was shown to improve the stability and bioavailability of drugs and ensure their safe application (29). The average nanomicelle is smaller than $100 \mathrm{~nm}$, which makes it suitable for in-vivo transfer and entering into the cells by endocytosis. The absorption pattern of endocytosis can 
A

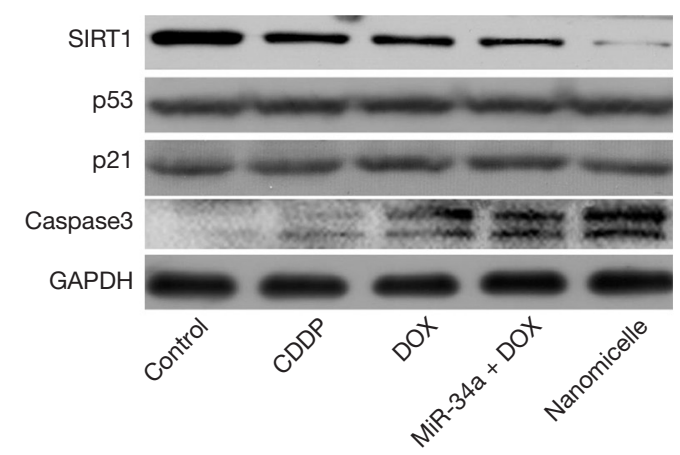

\section{B}

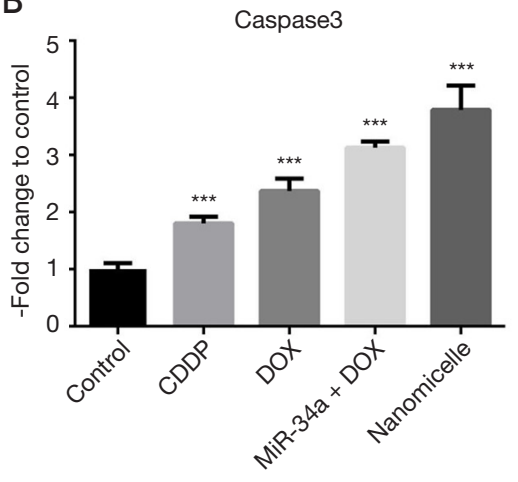

D

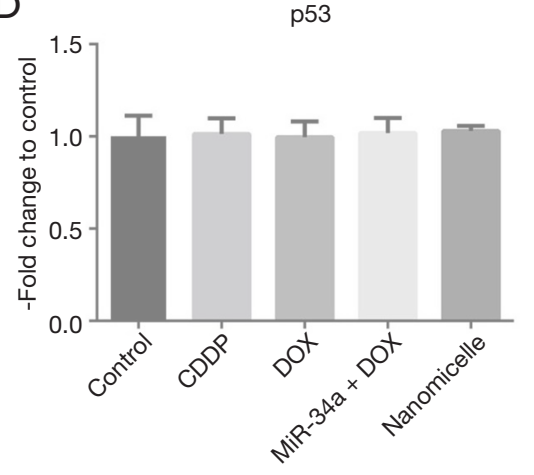

C

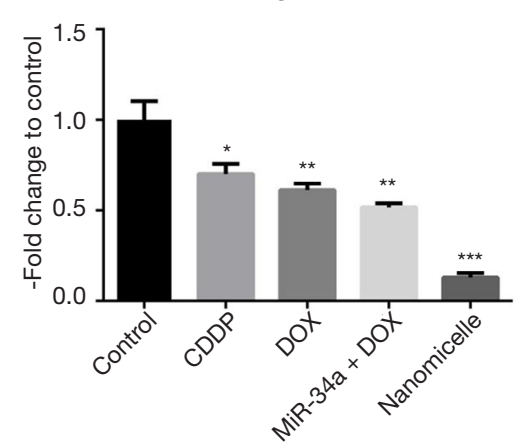

$\mathrm{E}$

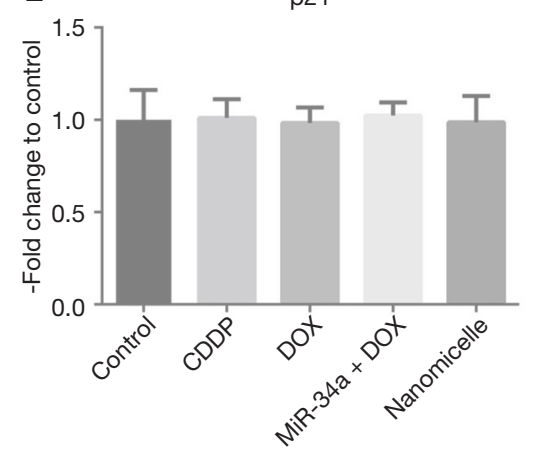

Figure 5 Nano-micelle increased the expression of SIRT1 by promoting the concentration of miR-34a in KYSE-410-CisR. (A) The expressions of SIRT1, p53, p21, caspase3, and GAPDH in KYSE-410-CisRs after incubation for $24 \mathrm{~h}$ with CDDP (6.524 $\mu \mathrm{g} / \mathrm{mL})$, DOX $(10 \mu M)$, miR-34a $(0.2 \mu M)+\operatorname{DOX}(10 \mu M)$, and nanomicelle (containing $10 \mu M$ DOX and $0.2 \mu M$ miR-34a mimic), respectively, detected by Western blot; (B) the gray value ratio of caspase3 versus GAPDH from five groups; (C) the gray value ratio of SIRT1 versus GAPDH from five groups; (D) the gray value ratio of 553 versus GAPDH from five groups; (E) the gray value ratio of p21 versus GAPDH from five groups. Three independent assays were performed $\left({ }^{*}, \mathrm{P}<0.05\right.$ vs. control; **, $\mathrm{P}<0.01$ vs. control; ${ }^{* *}, \mathrm{P}<0.001$ vs. control). CDDP, cisplatin; DOX, doxorubicin.

prevent drugs from being excreted by efflux transporters, such as P-gp (21). Thus, the ability of penetration into the tumor cells and retention in the tumor cells can be significantly improved. In this study, the nanomicelle comprised a mixture of $\mathrm{PEG}_{2 \mathrm{k}}-\mathrm{CLV}$-ADR, which contained PEG, a CLV with a high affinity to MMP2, and DOX. The mixture could promote the transferring of DOX in vivo and increase the retention interval and rate in the cell. A miR- 
34a-S-S-PE complex was also included in the nanomicelle, which is composed of a disulfide bond, miR-34a, and a phosphate ester (PE). Tumor cells containing large amounts of reduced glutathione (GSH) can degrade the disulfide bond, potentially releasing miR-34a. Simultaneously, PE can prevent miR-34a from degrading. The last component of the nanomicelle is the TAT-PEG $\mathrm{F}_{1 \mathrm{k}}-\mathrm{PE}$ complex, which is composed of a cysteine-modified TAT protein, $\mathrm{PEG}_{1 \mathrm{k}}$, and PE. The TAT-PEG ${ }_{1 k}-\mathrm{PE}$ complex can ensure that the nanomicelle is absorbed by cells via endocytosis. The results indicate that the nanomicelle could significantly increase the cell proliferation inhibition rate and apoptosis fraction of KYSE-410-CisR. The expression of miR-34a on a gene level and caspase 3 on a protein level in KYSE410-CisR was significantly increased after incubation with the nanomicelle. Meanwhile, the expression of SIRT1 was significantly downregulated. The nanomicelle promoted the anti-tumor effect of DOX by both activating the SIRT1 signal pathway and increasing the concentration of DOX in the tumor cells.

In summary, the specially designed nanomicelle demonstrated promising tumor cell proliferation inhibition and apoptosis-inducing properties by introducing miR-34a and enhancing the efficiency of DOX.

\section{Acknowledgments}

We would like to thank AME Editing Service for the English editing of this manuscript.

Funding: This work was supported by grants from the National Natural Science Foundation of China (Grant No. 81502647, 81502646), the Zhejiang Provincial Natural Science Foundation of China (Grant No. LY17H160039, LY18H160031, and LY19H160004), and the Zhejiang Medical and Health Science and Technology Project (Grant No. 2016KYA051, 2016146960,2018RC083, 2018260661, and2019328882).

\section{Footnote}

Conflicts of Interest: All authors have completed the ICMJE uniform disclosure form (available at http://dx. doi. org/10. 21037/tcr-19-975). The authors have no conflicts of interest to declare.

Ethical Statement: The authors are accountable for all aspects of the work in ensuring that questions related to the accuracy or integrity of any part of the work are appropriately investigated and resolved.

Open Access Statement: This is an Open Access article distributed in accordance with the Creative Commons Attribution-NonCommercial-NoDerivs 4.0 International License (CC BY-NC-ND 4.0), which permits the noncommercial replication and distribution of the article with the strict proviso that no changes or edits are made and the original work is properly cited (including links to both the formal publication through the relevant DOI and the license). See: https://creativecommons.org/licenses/by-nc-nd/4.0/.

\section{References}

1. Herszényi L, Tulassay Z. Epidemiology of gastrointestinal and liver tumors. Eur Rev Med Pharmacol Sci 2010;14:249-58.

2. Arnold M, Soerjomataram I, Ferlay J, et al. Global incidence of oesophageal cancer by histological subtype in 2012. Gut 2015;64:381-7.

3. Oppedijk V, van der Gaast A, van Lanschot JJ, et al. Patterns of recurrence after surgery alone versus preoperative chemoradiotherapy and surgery in the CROSS trials. J Clin Oncol 2014;32:385-91.

4. Sjoquist KM, Burmeister BH, Smithers BM, et al. Survival after neoadjuvant chemotherapy or chemoradiotherapy for resectable oesophageal carcinoma: an updated metaanalysis. Lancet Oncol 2011;12:681-92.

5. Blot WJ, Devesa SS, Kneller RW, et al. Rising incidence of adenocarcinoma of the esophagus and gastric cardia. JAMA 1991;265:1287-9.

6. Crane SJ, Richard Locke G 3rd, Harmsen WS, et al. The changing incidence of oesophageal and gastric adenocarcinoma by anatomic sub-site. Aliment Pharmacol Ther 2007;25:447-53.

7. Kort EJ, Sevensma E, Fitzgerald TL. Trends in esophageal cancer and body mass index by race and gender in the state of Michigan. BMC Gastroenterol 2009;9:47.

8. Derakhshan $M H$, Liptrot $S$, Paul J, et al. Oesophageal and gastric intestinal-type adenocarcinomas show the same male predominance due to a 17 year delayed development in females. Gut 2009;58:16-23.

9. Crane SJ, Locke GR 3rd, Harmsen WS, et al. Survival trends in patients with gastric and esophageal adenocarcinomas: a population-based study. Mayo Clin Proc 2008;83:1087-94.

10. Chen C, Ma Z, Zhang H, et al. Kruppel-like factor 4 enhances sensitivity of cisplatin to esophageal 
squamous cell carcinoma (ESCC) cells. Med Sci Monit 2017;23:3353-9.

11. Li DJ, Shi M, Wang Z. RUNX3 reverses cisplatin resistance in esophageal squamous cell carcinoma via suppression of the protein kinase B pathway. Thorac Cancer 2016;7:570-80.

12. Zhao Y, Ma K, Yang S, et al. MicroRNA-125a-5p enhances the sensitivity of esophageal squamous cell carcinoma cells to cisplatin by suppressing the activation of the STAT3 signaling pathway. Int J Oncol 2018;53:644-58.

13. Zhang P, Xi M, Li QQ, et al. Concurrent cisplatin and 5 -fluorouracil versus concurrent cisplatin and docetaxel with radiotherapy for esophageal squamous cell carcinoma: a propensity score-matched analysis. Oncotarget 2016;7:44686-94.

14. Shibata-Kobayashi S, Yamashita H, Okuma K, et al. Correlation among 16 biological factors [p53, p21(waf1), MIB-1 (Ki-67), p16(INK4A), cyclin D1, E-cadherin, Bcl2, TNF-alpha, NF-kappaB, TGF-beta, MMP-7, COX2, EGFR, HER2/neu, ER, and HIF-1alpha] and clinical outcomes following curative chemoradiation therapy in 10 patients with esophageal squamous cell carcinoma. Oncol Lett 2013;5:903-10.

15. Liu WK, Jiang XY, Zhang MP, et al. The relationship between HPV16 and expression of cyclooxygenase-2, P53 and their prognostic roles in esophageal squamous cell carcinoma. Eur J Gastroenterol Hepatol 2010;22:67-74.

16. Whibley C, Pharoah PD, Hollstein M. p53 polymorphisms: cancer implications. Nat Rev Cancer 2009;9:95-107.

17. Bunz F, Hwang PM, Torrance C, et al. Disruption of p53 in human cancer cells alters the responses to therapeutic agents. J Clin Invest 1999;104:263-9.

18. Tung MC, Lin PL, Wang YC, et al. Mutant 553 confers chemoresistance in non-small cell lung cancer by upregulating Nrf2. Oncotarget 2015;6:41692-705.

19. Porschen R, Classen S, Piontek M, et al. Vascularization of carcinomas of the esophagus and its correlation with tumor proliferation. Cancer Res 1994;54:587-91.

Cite this article as: Fang J, Wang Y, Wang Z, Xie T, Yan F, Wang L, Hua J, Wang F, Fu Z, Ye Z. A nanomicelle with miR$34 \mathrm{a}$ and doxorubicin reverses the drug resistance of cisplatin in esophageal carcinoma cells by inhibiting SIRT1 signal pathway. Transl Cancer Res 2020;9(7):4131-4140. doi: 10.21037/tcr-19-975
20. Li W, Zhang H, Assaraf YG, et al. Overcoming ABC transporter-mediated multidrug resistance: Molecular mechanisms and novel therapeutic drug strategies. Drug Resist Updat 2016;27:14-29.

21. Lu J, Zhao W, Huang Y, et al. Targeted delivery of Doxorubicin by folic acid-decorated dual functional nanocarrier. Mol Pharm 2014;11:4164-78.

22. Ye ZM, Dai SJ, Yan FQ, et al. DCE-MRI-derived volume transfer constant (Ktrans) and DWI apparent diffusion coefficient as predictive markers of short- and long-term efficacy of chemoradiotherapy in patients with esophageal cancer. Technol Cancer Res Treat 2018;17:1533034618765254.

23. Ye Z, Fang J, Dai S, et al. Inter- and intra-observer reproducibility of ADC measurements in esophageal carcinoma primary tumors. Oncotarget 2017;8:92880-9.

24. Ye Z, Fang J, Dai S, et al. MicroRNA-34a induces a senescence-like change via the down-regulation of SIRT1 and up-regulation of $\mathrm{p} 53$ protein in human esophageal squamous cancer cells with a wild-type p53 gene background. Cancer Lett 2016;370:216-21.

25. Xiao Z, Li CH, Chan SL, et al. A small-molecule modulator of the tumor-suppressor miR34a inhibits the growth of hepatocellular carcinoma. Cancer Res 2014;74:6236-47.

26. Dorn GW 2nd. miR-34a and the cardiomyopathy of senescence: SALT PNUTS, SALT PNUTS! Cell Metab 2013;17:629-30.

27. Raver-Shapira N, Marciano E, Meiri E, et al. Transcriptional activation of miR-34a contributes to p53mediated apoptosis. Mol Cell 2007;26:731-43.

28. Shi H, Zhou S, Liu J, et al. miR-34a inhibits the in vitro cell proliferation and migration in human esophageal cancer. Pathol Res Pract 2016;212:444-9.

29. Salzano G, Costa DF, Sarisozen C, et al. Mixed nanosized polymeric micelles as promoter of doxorubicin and miRNA-34a co-delivery triggered by dual stimuli in tumor tissue. Small 2016;12:4837-48. 